\title{
The use of DTW method as an effective way of uroflowmetry data screening analysis
}

\author{
Wojciech Walendziuk ${ }^{1}$, Aleksander Sawicki ${ }^{2}$, Adam Idźkowski ${ }^{3}$ \\ $1,2,3$ Bialystok University of Technology, Poland \\ E-mails: ${ }^{1}$ w.walendziuk@pb.edu.pl (corresponding author), ${ }^{2}$ aleksander.sawicki.91@ gmail.com, \\ ${ }^{3}$ a.idzkowski@pb.edu.pl \\ (Received 29 March 2016; accepted 29 June 2016)
}

\begin{abstract}
The aim of this work is to present an application of the Dynamic Time Warping (DTW) method for the preliminary classification of data obtained during uroflowmetric tests. This enables determining whether the recorded data from the urine flow speed measurements is accurate or not. Example urine flow characteristics obtained from a uroflowmeter based on a strain gauge transducer were used in the research. The analysis of the algorithm performance was done on the basis of real tests results of patients with the risk of the prostate hyperplasia occurrence. Moreover, the results of example experiments are presented in this paper.
\end{abstract}

Keywords: uroflowmeter, uroflowmetry, urine flow, dynamic time warping.

\section{Introduction}

The benign prostatic hyperplasia is one of most frequent diseases occurring at men over 50 years old. The enlargement of the prostate gland $(>30 \mathrm{ml})$ is the reason of lesions within the urinary system, called the acute urinary retention (AUR) [1]. One of the ways of patient examination is uroflowmetry which can be treated as a non-invasive preliminary diagnostic method. Currently the devices used to measure the urine flow are usually based on strain gauge sensors [2-5]. It is possible to determine the urine mass by the voltage analysis in bridge circuits (e.g. Wheatstone's bridge). Additionally, recording data with a defined sampling frequency, it is possible to determine the urine mass increase in time. A flowmeter equipped with a rotating disc on which the patient's urine drops is another solution [2]. The disc rotates with constant speed due to an electric engine. In a measurement system of this type, the values such as current voltage supplying the engine, which is proportional to the urine flow intensity, are measured. The urine flow speed measurement by analyzing the hydrostatic pressure read by a pressure transducer is another solution. Such system uses the pressure measurement in a container closed at one side, e.g. at the top, where a pressure sensor is placed. The open side is situated in a container with fluid, therefore, raising its level causes the pressure increase. The pressure is, then, proportional to the urine flow [5]. All systems enable estimating the urine flow through the urethra on the basis of the measurements of the excreted urine volume and its flow speed [6-8]. In tests of this type, micturition parameters are usually presented in the form of a graph. Evaluation of the parameters helps the urologist to plan the therapy and monitor the conservative treatment.

Unfortunately, the lack of possibility of automatic data analysis in order to identify the abnormalities in the urine flow is a disadvantage of this solution. There are, however, devices that analyze the urine flow characteristics by applying the neural network, but they require appropriate computing performance. For all those reasons, the main aim of this work is to create an algorithm of the measurement data analysis which could be helpful with the BPH (benign prostatic hyperplasia) preliminary diagnosis. This algorithm could be implemented in a mobile uroflowmeter which would draw the urine flow characteristics, whose analysis would be used to classify patients to further diagnostic tests. The idea could also be used in a device permanently installed in the patient's house. The tests concerning the benign prostatic hyperplasia could be

(C) 2016 The Authors. Published by VGTU Press. This is an open-access article distributed under the terms of the Creative Commons Attribution License (CC-BY 4.0), which permits unrestricted use, distribution, and reproduction in any medium, provided the original author and source are credited. 
carried out constantly, and the device could inform the patient about the necessity of consulting the doctor immediately after detecting the urine flow disorder.

\section{DTW algorithm as a method of uroflowmetric data analysis}

The principle of the DTW algorithm is to compare a series of measurement results that have common features but are different in terms of time and amplitude [9].

The algorithm of comparing the flows in time uses two signals: the model one $-X_{p}$, and the measured one $-X_{m}$, whose individual samples are recorded in the data board. During the first stage of the algorithm work, Euclidean distances between all samples of the compared signals are determined (1):

$$
Y\left(n_{p}, n_{m}\right)=\sqrt{\sum_{i=1}^{N_{p} \times N_{m}}\left(X_{p}(i)-X_{m}(i)\right)^{2}}, n_{p}=1 \ldots N_{p}, n_{m}=1 \ldots N_{m} .
$$

Next, the $Y_{a c}$ cumulative cost on the matrix boundary elements towards $X_{p}$ and $X_{m}$ is calculated. Searching the path of the least cumulative cost of transition will be the next step of the process (2). In order to do it, the backtracking procedure is used. It consists in transition from the $\left(N_{p}, N_{m}\right)$ point to $(1,1)$. Minimizing the transition function, e.g. finding the path in the matrix that has the least cumulative cost, is an important element of this stage of the process. The function that describes this issue can be presented as:

$Y_{c}=\sum_{i=N_{p}}^{1} \sum_{j=N_{m}}^{1} \min \left\{Y_{a c}(i-1, j-1) ; Y_{a c}(i, j-1) ; Y_{a c}(i-1, j)\right.$

After finding the shortest path, the elements qualified as the least are summed up.
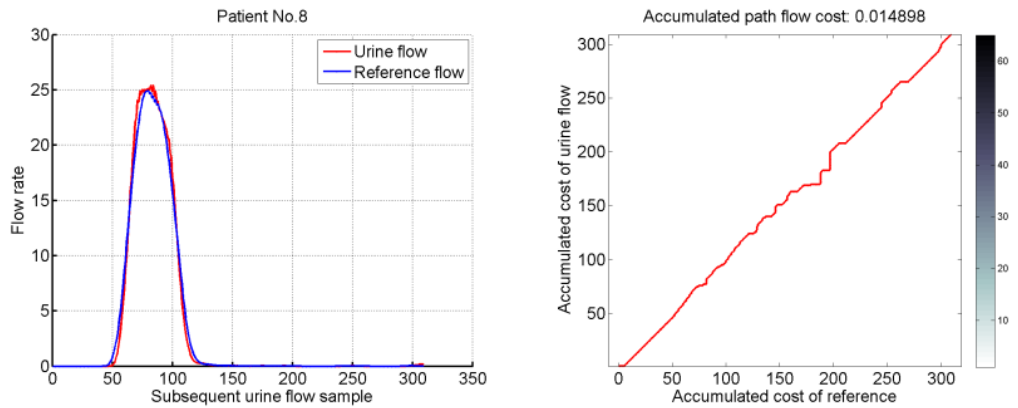

Fig. 1. Proper urine flow and the DTW least cost path (0.01489)
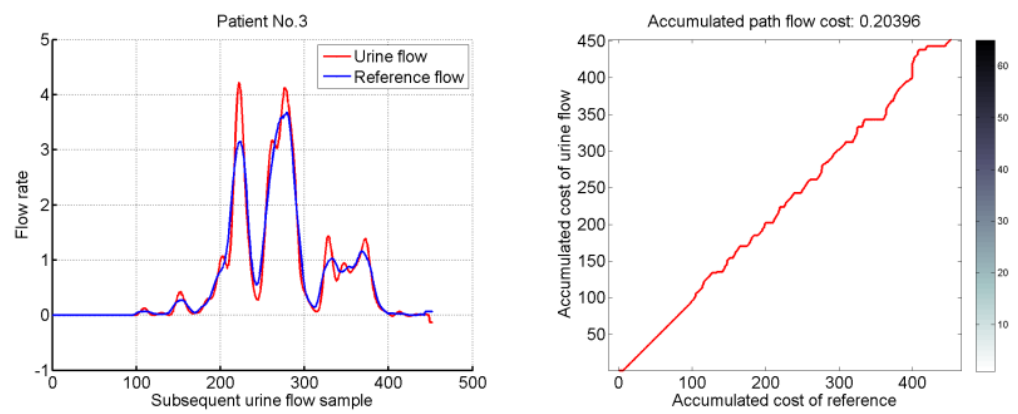

Fig. 2. Improper urine flow and the DTW least cost path (0.20396) 
Those summed up numerical values can be then used as a classifier of the measurement data analysis, indicating the degree of the signals similarity. It is worth stressing that in the carried out experiments, the average value of the urine flow obtained during the measurement was used as the reference model. Due to this fact, the algorithm employing the DTW method was not sensitive to the urination time, the urine flow amplitude or the urination initiation time until the moment of the maximum value of the urine flow. An example of the accurate urine flow and the characteristics of the transition path with the accumulative cost are presented in Fig. 1. Whereas, the inaccurate urine flow can be seen in Fig. 2.

In order to test the algorithm, an original uroflowmeter based on a strain gauge was used [4]. The device was equipped with a microcontroller that acquired the measurement data and recorded it in the local memory. After the tests had been done, their analysis was conducted with the use of the previously mentioned DTW algorithm in the MATLAB environment. A group of randomly chosen 20 patients aged 45-82 with hypothetic prostate hyperplasia were tested for the purpose of this research. The obtained results were consulted with a urologist in terms of the correctness of their medical interpretation.

As a result of implementing the presented algorithm, satisfying data analysis results were obtained. In the tested cases, the following cost coefficients were applied: less than 0.3 for the accurate flows and more than 0.31 for the inaccurate flows. The efficiency of detecting the accurate urine flows equaled $97 \%$.

\section{Conclusions}

The present work contains the implementation of the DTW method in the urine flow characteristics analysis. Real results of the uroflowmetric tests of a group of men aged 45-82 with hypothetic prostate hyperplasia were used in the research. The tests of the algorithm proved its correctness of $97 \%$, which can be treated as a very satisfactory result. Such a great efficiency caused the authors to implement the worked out algorithm in a microcontroller-based measurement system which will acquire data from a strain gauge transducer. Further research will be therefore focused on an algorithm of the reference flow automatic generating, as well as on constructing a new version of the device aiming at measuring the urine flow speed.

\section{Acknowledgements}

The paper was prepared at Bialystok University of Technology within a framework of the S/WE/1/2013 and the MB/WE/4/2016 projects sponsored by Ministry of Science and Higher Education.

\section{References}

[1] Marks, L. S.; Roehrborn, C. G.; Andriole, G. L. 2006. Prevention of benign prostatic hyperplasia disease, Journal of Urology 176(4): 1299-1306. http://dx.doi.org/10.1016/j.juro.2006.06.022

[2] Dejhan, R. B. K.; Yimman, S. 2014. Uroflowmetry recording design, in Proceedings of TENCON 2014 - IEEE Region 10 Conference, 22-25 October 2014, Bangkok, Thailand. Piscataway: IEEE. http://dx.doi.org/10.1109/TENCON.2014.7022392

[3] Makal, J.; Idźkowski, A.; Walendziuk, W. 2006. Computer assisted uroflowmetry diagnostic system, Proceedings of SPIE 6347: 63472B. http://dx.doi.org/10.1117/12.714637

[4] Walendziuk, W.; Idzkowski, A. 2009. Portable acquisition system for domiciliary uroflowmetry, Journal of Vibroengineering 11(3): 592-596.

[5] Suryawanshi, A.; Joshi, A. 2012. Urine flow rate measurement based on volumetric pressure measurement principle, in 1st International Symposium on Physics and Technology of Sensors, 7-10 March 2012, Pune, India. http://dx.doi.org/10.1109/ISPTS.2012.6260961

[6] Altunay, S., et al. 2006. Interpretation of uroflow graphs with artificial neural networks, in Proceedings of IEEE Signal Processing and Communications Applications, 16-19 April 2006, Antalya, Turkey. Piscataway: IEEE. http://dx.doi.org/10.1109/SIU.2006.1659698 
[7] Chapple, C. R.; Mangera, A. 2011. Overview of the evaluation of lower urinary tract dysfunction, in Practical Urology: Essential Principles and Practice. Ed. by C. R. Chapple, W. D. Steers. London: Springer, 261-282. http://dx.doi.org/10.1007/978-1-84882-034-0_20

[8] Bray, A., et al. 2012. Methods and value of home uroflowmetry in the assessment of men with lower urinary tract symptoms: a literature review, Neurourology and Urodynamics 31(1): 7-12. http://dx.doi.org/10.1002/nau.21197

[9] Müller, M. 2007. Information Retrieval for Music and Motion. Berlin Heidelberg: Springer-Verlag. 318 p. http://dx.doi.org/10.1007/978-3-540-74048-3 